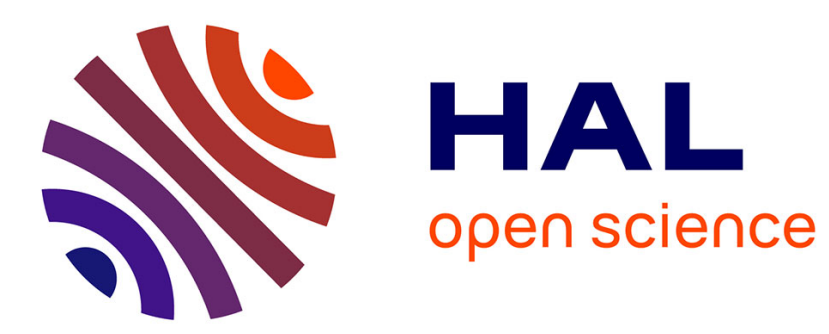

\title{
Description d'un anémomètre doppler-laser destiné à l'étude de la turbulence dans les fluides \\ G. Gouesbet, M. Trinité
}

\section{To cite this version:}

G. Gouesbet, M. Trinité. Description d'un anémomètre doppler-laser destiné à l'étude de la turbulence dans les fluides. Revue de Physique Appliquée, 1974, 9 (3), pp.571-574. 10.1051/rphysap:0197400903057100 . jpa-00243820

HAL Id: jpa-00243820 https://hal.science/jpa-00243820

Submitted on 1 Jan 1974

HAL is a multi-disciplinary open access archive for the deposit and dissemination of scientific research documents, whether they are published or not. The documents may come from teaching and research institutions in France or abroad, or from public or private research centers.
L'archive ouverte pluridisciplinaire HAL, est destinée au dépôt et à la diffusion de documents scientifiques de niveau recherche, publiés ou non, émanant des établissements d'enseignement et de recherche français ou étrangers, des laboratoires publics ou privés. 
Classification

Physics Abstracts

$0.640-6.315$

\title{
DESCRIPTION D'UN ANÉMOMẼTRE DOPPLER-LASER DESTINÉ A L'ÉTUDE DE LA TURBULENCE DANS LES FLUIDES
}

\author{
G. GOUESBET et M. TRINITÉ \\ Laboratoire de Thermodynamique, E. R. A. du C. N. R. S. $n^{0} 254$ \\ Faculté des Sciences et des Techniques de Rouen \\ 76130 Mont-Saint-Aignan, France
}

(Reçu le 2 janvier 1974)

\begin{abstract}
Résumé. - Nous présentons un anémomètre Doppler à laser, en montage interférentiel, avec analyse du signal par échantillonnage. Nous effectuons une double validation des valeurs de l'échantillon : validation électronique par une " plaquette logique » détectant des " mauvais signaux », validation informatique au niveau du programme de calcul. Nous comparons nos résultats expérimentaux à ceux de J. A. Clark, Laufer J. et Comte-Bellot.
\end{abstract}

\begin{abstract}
A Doppler-laser anemometer, with a two-beam, dual-scatter system technique, and using a signal sample, is described. Sample values are validated electronically by using a detector of bad signals ; addition verification is obtained with a computing programme. Data are compared with those of J. A. Clark, J. Laufer and Comte-Bellot.
\end{abstract}

1. Montage optique (Fig. 1). - La source lumineuse est un laser $\mathrm{He}-\mathrm{Ne}$, puissance $5 \mathrm{~mW}$, travaillant dans le mode $\mathrm{TEM}_{00}$, à $6328 \AA$. Le séparateur de faisceaux SF fournit deux faisceaux parallèles, d'intensités sensiblement égales, que la lentille $\mathrm{L}_{1}$ fait converger dans le fluide en $M$, où est créé un champ d'interférences tridimensionnel d'interfrange $i=1,61 \mu$. Une particule présente dans le fluide, supposée se mouvant à la même vitesse $\mathbf{u}$, que lui, traversant le champ $M$, et de diamètre $\varphi_{\mathrm{p}}<i$, diffuse la lumière incidente. La lumière diffusée est modulée à la fréquence

$$
f=|\mathbf{u} \cdot \mathbf{n}| / i,
$$

n étant le vecteur unitaire perpendiculaire à la direction des franges. L'axe optique étant perpendiculaire à l'axe du tube de verre dans lequel s'écoule le fluide, on a, en désignant par $u$ la composante axiale de $\mathbf{u}$ :

$$
|\mathbf{u} \cdot \mathbf{n}|=u \text { et } f_{\mathrm{MHz}}=u_{\mathrm{cm} / \mathrm{s}} / 161 \text {. }
$$

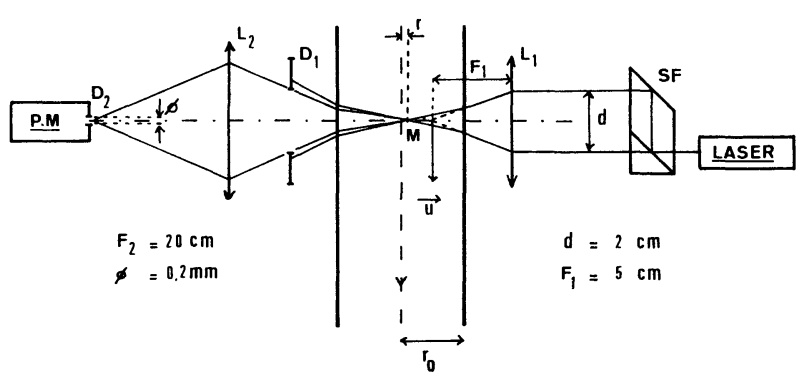

FIG. 1. - Montage optique.
Un diaphragme $D_{1}$ arrête les faisceaux transmis. Une lentille $\mathrm{L}_{2}$ conjugue le champ $M$ et le diaphragme $\mathrm{D}_{2}$ d'entrée du photomultiplicateur EMI 9558 avec un grandissement de l'ordre de 4 . Le photomultiplicateur transforme la lumière diffusée modulée à la fréquence $f$ en un signal électronique de fréquence $f$.

Ce montage optique peut être interprété par la théorie de l'effet Doppler, justifiant la dénomination de cette anémométrie.

La résolution spatiale [3] de cette méthode locale dépend des dimensions et de la forme du champ $M$. Ce domaine de mesures est un ellipsoïde, sensiblement de révolution, dont le petit axe vaut $2 a \sim 60 \mu$ et le grand axe $2 c \sim 400 \mu$. Le volume en est $\sim 0,8 \times 10^{-6} \mathrm{~cm}^{3}$. Le nombre de franges présentes est $\mathcal{N} \sim 38$ franges.

2. Montage électronique (Fig. 2). - Le signal électronique issu du photomultiplicateur est filtré, et amplifié suffisamment pour pouvoir être traité par la chaîne de mesures : il a la forme donnée par la figure 3 pour le passage d'une seule particule dans le domaine de mesures. La modulation en amplitude provient de la répartition gaussienne d'énergie dans un faisceau laser $\mathrm{TEM}_{00}$. Le signal est également modulé en fréquence [4] par :

- élargissement géométrique dû à l'angle solide des faisceaux laser incidents, estimé par $\left(\sigma_{f, \text { géom }} / f\right)=2,8 \%$,

- élargissement naturel dû au temps de transit fini d'une particule diffusante dans le domaine $M$, estimé $\operatorname{par}\left(\sigma_{f, \text { nat }} / f\right)=1,1 \%$, 


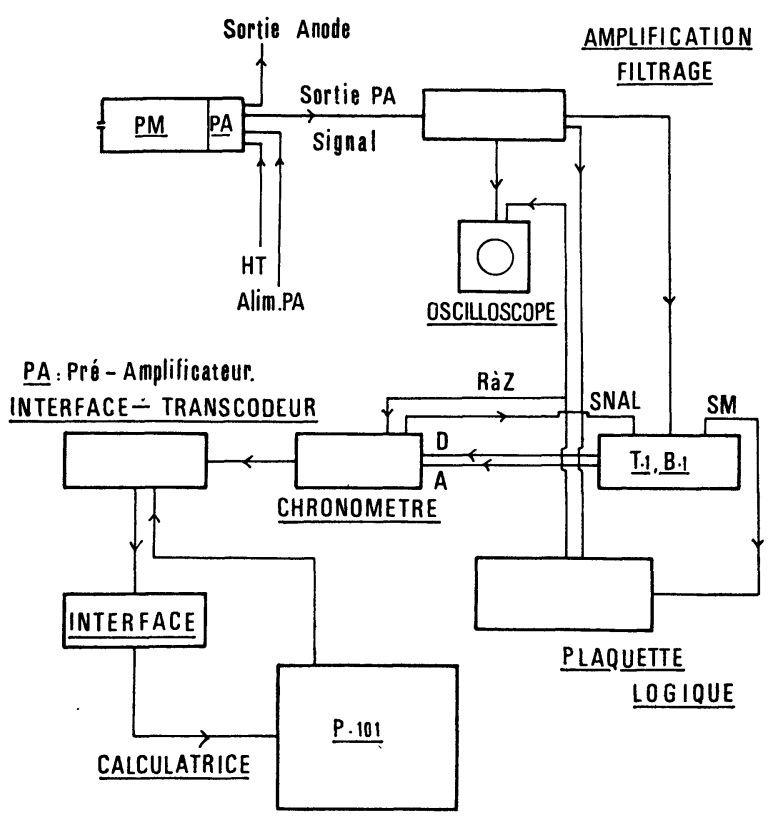

FIG. 2. - Montage électronique.

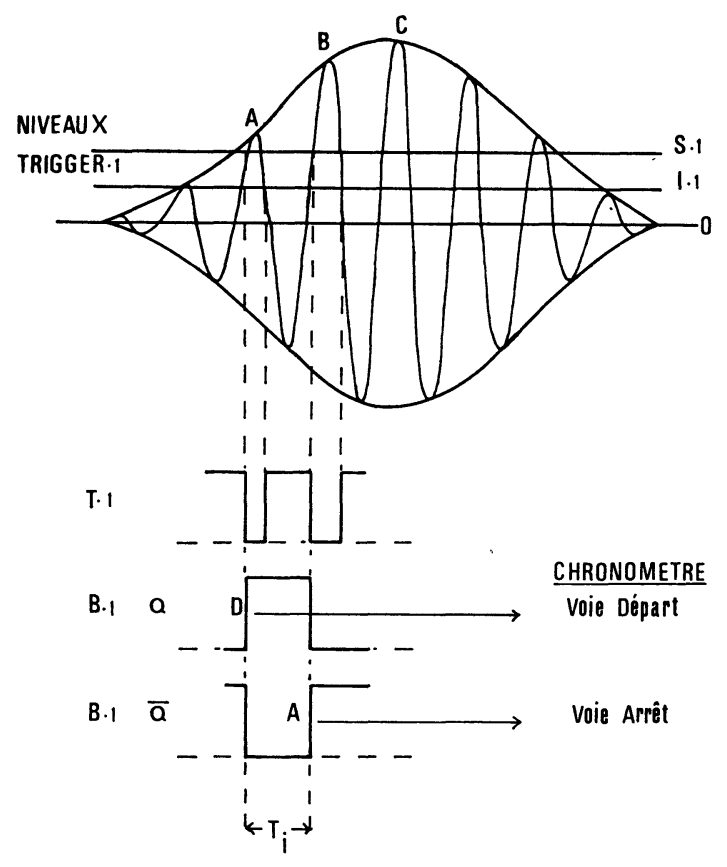

Fig. 3. - Signal et mise en forme.

- élargissement de gradient dû au gradient de vitesse dans le domaine $M$, estimé par

$$
\left(\sigma_{f, \text { grad }} / f\right) \%=(50 \mathrm{~cd} u / u \mathrm{~d} r),
$$

- élargissement de turbulence dû aux fluctuations de vitesse, donné par $\left(\sigma_{f, \text { turb }} / f\right)=\left(\sigma_{u, \text { turb }} / \bar{u}\right)$.

Le signal est mis en forme par le trigger $\mathrm{T} 1$ suivi de la bascule B 1 (Fig. 3). Le chronomètre utilisé est un CHN 12-M 1 des poudreries du Bouchet (Résolution $20 \mathrm{~ns})$. La voie Départ est déclenchée par le front de montée $\mathrm{D}$, la voie Arrêt par le front de montée A.
On mesure ainsi une période $T_{i}$. Les calculs sont effectués sur un échantillon de $N$ valeurs $T_{i}$, grâce à une chaîne automatique d'acquisition et de calcul des données qui sera décrite en [10].

3. Validation électronique. - Détection de " mauvais signaux " par une "plaquette logique".

Si l'amplitude de l'alternance positive B (Fig. 3) devient inférieure au niveau $S 1$ du trigger $T$, alors que les amplitudes des alternances positives $\mathrm{A}$ et $\mathrm{C}$ lui sont supérieures, c'est que le signal est déformé. Un tel " mauvais signal " impliquera une mesure égale à $(2 T)_{i}$. De même, le chronomètre pourra effectuer des mesures $(3 T)_{i},(4 T)_{i}$, etc. Nous disposons ainsi d'un moyen simple et peu coûteux de reconnaître des

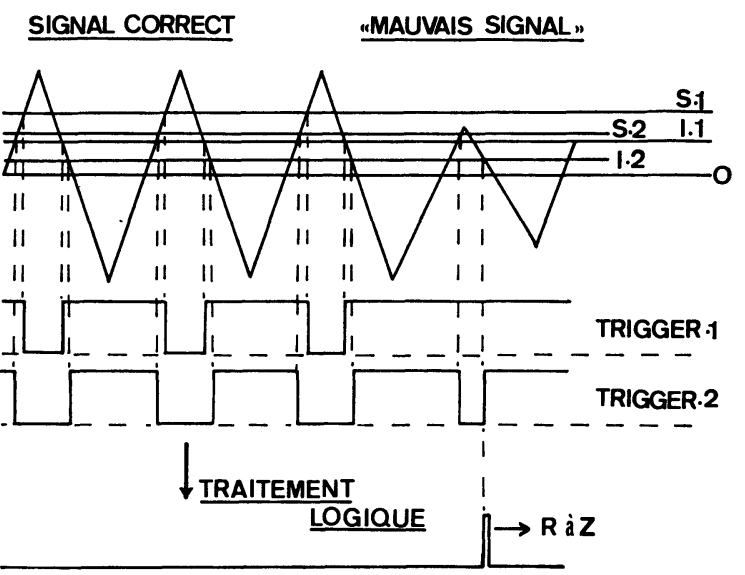

Fig. 4. - Principe de la «plaquette logique »

" mauvais signaux ». La " plaquette logique " permet de les détecter. Le principe en est fourni figure 4. Un trigger $\mathrm{T} 2$ a des niveaux $\mathrm{S} 2$ et $\mathrm{I} 2$ inférieurs respectivement aux niveaux $\mathrm{S} 1$ et $\mathrm{I} 1$ du trigger $\mathrm{T} 1$. A l'apparition d'un " mauvais signal », le trigger T 2 passe du niveau logique 1 au niveau 0 , alors que le trigger $\mathrm{T} 1$ reste sur le niveau 1 . La " plaquette logique » utilise cette différence et fournit une impulsion $(R$ à $Z)$ de Remise à Zéro en cours de lecture d'une « mauvaise valeur ", la mesure n'étant pas alors terminée, et aucune valeur n'étant prise en compte par la chaîne automatique d'acquisition et de calcul des données. Les signaux SM (Signal Mesure) et SNAL (Signal " Niveaux Attente-Lecture ") apparaissant sur la figure 2 sont nécessaires au fonctionnement de la " plaquette logique ». Ce fonctionnement sera décrit plus complètement en [10].

4. Validation informatique. - L'efficacité de la « plaquette logique » n'est pas totale, certaines valeurs multiples de $T_{i}$ étant encore transférées en sortie. Le programme de calcul est conçu pour éliminer les erreurs qui pourraient résulter de la prise en compte d'une valeur erronée. Les 20 premières valeurs reconnues correctes fournissent une estimation $\bar{f}_{0}$ de la fré- 
quence moyenne. Les valeurs suivantes sont comparées aux valeurs

$$
f_{\max }=\bar{f}_{0}+0,4 \bar{f}_{0} \quad \text { et } \quad f_{\min }=\bar{f}_{0}-0,4 \bar{f}_{0} .
$$

Le coefficient 0,4 est choisi sur des critères statistiques : pour un écart quadratique de $17 \%$ environ, et supposant une turbulence gaussienne, deux valeurs correctes environ sur 100 seront éliminées de ce fait. Les valeurs en dehors de l'intervalle $\left(f_{\max }, f_{\min }\right)$ sont rejetées. Les 50 premières valeurs suivantes acceptées fournissent une estimation $\bar{f}_{1}$ de la fréquence moyenne et $\left(\sigma_{f}\right)_{1}$ de l'écart quadratique. Les valeurs suivantes sont comparées à l'intervalle

$$
\left(\bar{f}_{1}+2,3\left(\sigma_{f}\right)_{1}, \bar{f}_{1}-2,3\left(\sigma_{f}\right)_{1}\right) \text {. }
$$

Le coefficient 2,3 est choisi de sorte que seulement deux valeurs correctes sur 100 environ seront éliminées de ce fait. Les 50 valeurs suivantes acceptées fournissent les estimations $\bar{f}_{2}$ et $\left(\sigma_{f}\right)_{2}$, et l'intervalle de validation $\left(\bar{f}_{2}+2,3\left(\sigma_{f}\right)_{2}, f_{2}-2,3\left(\sigma_{f}\right)_{2}\right)$. Les $N$ premières valeurs suivantes acceptées constituent l'échantillon définitif, et fournissent les estimations $\bar{f}$ de la fréquence moyenne, et $\left(\sigma_{f, \text { tot }} \mid \bar{f}\right) \mathrm{du}$ coefficient de variation.

5. Résultats expérimentaux. - 5.1 L'ÉCOULEMENT. - Le fonctionnement de l'anémomètre est testé par l'étude d'un écoulement turbulent établi d'eau produit dans un tube de verre cylindrique, de section circulaire (rayon intérieur: $15 \mathrm{~mm}$ ), sous l'influence d'une charge constante. Le fluide est ensemencé en particules diffusantes artificielles (100 ppm de lait). Les mesures sont effectuées pour un nombre de Reynolds de $10^{4}$.

5.2 Mesures. - Les vitesses moyennes $u$ mesurées sont comparées aux résultats de Laufer [5] (Fig. 5). Les échantillons sont de taille 200 pour les points où les intensités de turbulence ont été également mesurées, et de taille 50 pour les autres. Le léger désaccord observé près de la paroi peut aisément s'interpréter par une erreur sur la localisation du champ d'interférences.

Les intensités de turbulence $\left(\sigma_{u, \text { turb }} / \bar{u}\right) \%$ sont déduites des coefficients de variation $\left(\sigma_{f, \text { tot }} / \bar{f}\right) \%$ par la relation :

$$
\begin{aligned}
\left(\sigma_{f, \text { tot }} / \bar{f}\right)^{2}=\left(\sigma_{u, \text { turb }} / \bar{u}\right)^{2}+ & \left(\sigma_{f, \text { géom }} / f\right)^{2}+ \\
& +\left(\sigma_{f, \text { nat }} / f\right)^{2}+\left(\sigma_{f, \text { grad }} / f\right)^{2} .
\end{aligned}
$$

Les résultats expérimentaux sont portés en intensités de turbulence rapportées à la vitesse sur l'axe (Fig. 5) et en $\left(\sigma_{u} / u^{*}\right)=f\left[u^{*}\left(r_{0}-r\right) / v\right]$ sur la figure 6. La vitesse de frottement $u^{*}=\left(\tau_{0} / \rho\right)^{1 / 2}$ est déduite de la mesure du débit à l'aide de la relation :

$$
\tau_{0}=0,03955 \rho v_{\mathrm{q}}^{7 / 4} v^{1 / 4}\left(2 r_{0}\right)^{-1 / 4}
$$

$v_{\mathbf{q}}$ étant la vitesse moyennée spatialement sur la section du tube de verre. Ces résultats sont comparés avec ceux de J. A. Clark [6], Laufer J. [7] et Comte-

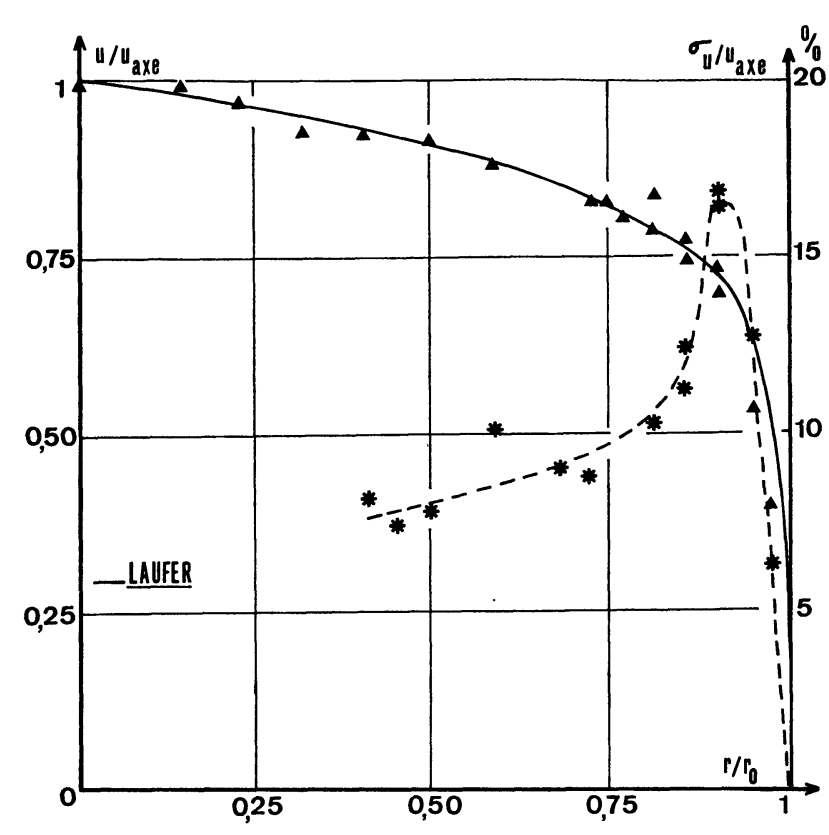

Fig. 5.

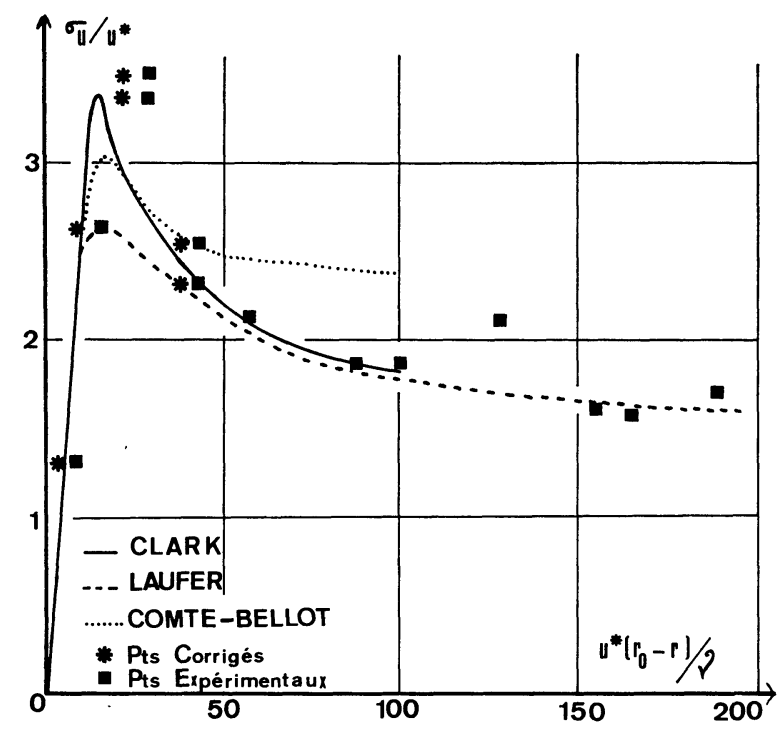

FIG. 6.

Fig. 5 et $6 .-$ Résultats expérimentaux.

Bellot [8] (Fig. 6). Sur cette figure 6, nos résultats expérimentaux sont représentés par les carrés noirs. Près de la paroi, nous avons apporté une correction relative à la position de ces points, dont le principe relève des considérations suivantes :

Laufer a utilisé un fil chaud. S'il n'est pas exclu que de ce fait, la contribution à $\sigma_{u}$ des composantes de plus hautes fréquences lui ait échappé, on peut, par contre, accorder un très grand crédit au profil des vitesses moyennes qu'il a obtenu (Fig. 5). Par notre dispositif, les mesures de $u$ et $\sigma_{u}$ sont obtenues simultanément pour le même point de l'espace exploré. La position de ce point peut être alors définie par la distance à la 
paroi qui, sur le profil des vitesses moyennes de Laufer, correspond à la vitesse moyenne mesurée. Nous obtenons ainsi les points corrigés (étoiles) sur la figure 6 . Nos résultats s'accordent alors remarquablement avec ceux de J. A. Clark. Cependant les expériences de ce dernier ont été réalisées dans une veine rectangulaire.

Enfin, les moments statistiques des $3^{\mathrm{e}}$ et $4^{\mathrm{e}}$ ordres peuvent être calculés sur les échantillons.

\section{Bibliographie}

[1] Yeh, Y. and Cummins, H. Z., Appl. Phys. Lett. 4 (1964) 176-178.

[2] Foreman, J. W., Jr., George, E. W. and Lewis, R. D., Appl. Phys. Lett. 7 (1965) 77-78.

[3] Lectures séries 39. Laser Technology in Aerodynamic Measurements. June 14-18. 1971. Von Karman Institute for Fluid Dynamics T 1 et $\mathrm{T} 2$.

[4] Mazumier, M. K. and Wankum, D. L., IEEE J. Quantum Electron. (1969) pp. 316-318.
[5] Schlichting, Boundary layer theory. Chapitre XX: turbulent flow through pipes (Mc Graw-Hill).

[6] Clark, J. A., Trans. ASME Ser. D. 90 (1968) 455.

[7] LAUfER, J., NACA Tech. Repts 1174, 1954, no 1174.

[8] Сомте-Bellot, G., Publications Scientifiques et Techniques du Ministère de l'Air (1965) $\mathrm{n}^{\circ} 419$.

[9] Gouesbet, G., Thèse de 3e cycle, Rouen 15 mars 1973.

[10] GouesBet, G., Etude de la turbulence dans les fluides par anémométrie Doppler-Laser interférentielle, avec échantillonnage du signal. A paraître 